\title{
Avaliação da atividade física de pacientes no pré-operatório de cirurgia cardíaca
}

\section{Evaluation of physical activity of patients in the preoperative period of cardiac surgery}

\author{
Priscila Cavalcante Sá, Nayan Leonardo Sousa Lopes, \\ Nathália Lima Costa, Marianne Lucena da Silva, \\ Rodolfo Gomes do Nascimento, Katiane da Costa Cunha
}

Como citar este artigo: SÁ, PRISCILA C.; LOPES, NAYAN L. S.; COSTA, NATHÁLIA L.; DA SILVA, MARIANNE L.; NASCIMENTO, RODOLFO G. CUNHA, KATIANA DA C. Avaliação da atividade física de pacientes no pré-operatório de cirurgia cardíaca. Revista Saúde (Sta. Maria). 2020; 46 (2).

\section{Autor correspondente:}

Nome: Marianne Lucena da Silva E-mail: mariannebsb@gmail.com

Formação Profissional:

Universidade Federal de Jataí,

Curso de Fisioterapia

Filiação Institucional: Universidade Federal de Jatai

Endereço para correspondência: Câmpus Jatobá - Cidade Universitária BR 364, km 195, nº 3800

Cidade: Jataí

Estado: Goiás

CEP: $75801-615$

Data de Submissão:

26/04/2020

Data de aceite:

28/08/2020

Conflito de Interesse: Não há conflito de interesse

\section{(cc) $\mathrm{BY}-\mathrm{NC}-\mathrm{ND}$}

\section{RESUMO}

Introdução: As doenças cardiovasculares (DCVs) lideram o ranking de causas de mortes no Brasil e no mundo. No início do século XX, eram responsáveis por $10 \%$ das mortes mundiais, porém, no ano de 2013, já correspondiam a 31,5\%. No Brasil, dados da Organização Mundial de Saúde (OMS), apontam que as DCVs causaram 31\% das mortes no país em 2014, seguindo a média mundial. Objetivo: avaliar o nível de atividade física de pacientes no pré-operatório de cirurgia cardíaca através da aplicação do questionário. Métodos: 0 questionário de Baecke foi utilizado como instrumento de pesquisa em 20 pacientes cardiopatas, referidos para cirurgia de forma eletiva, com faixa etária igual ou acima de 30 anos, de ambos os sexos. Analisou-se o nível de atividade física destes pacientes a partir das respostas que deram para duas variáveis: Exercícios Físicos de Lazer (EFL); e Atividades de Lazer e Locomoção (ALL). A avaliação dessas variáveis quantitativas foi realizada pela correlação de Pearson. Como resultado, percebeu-se que $60 \%$ dos pacientes eram do sexo masculino, com idade média de $53 \pm 10$ anos. A avaliação da interdependência entre os indicadores EFL e ALL realizada pela Correlação linear de Pearson resultou no $p$-valor $<0.0001$, o qual é altamente significante coeficiente de Correlação entre $r=0.8648$, indicando forte correlação entre o EFL e o ALL. Conclusão: Pacientes em pré-operatório de cirurgia cardíaca são em sua maioria homens acima dos 50 anos que possuem um nível de condicionamento físico bom.

PALAVRAS-CHAVE: Atividade motora; Cardiopatias; Perfil de saúde.

\section{ABSTRACT}

Introduction: Cardiovascular diseases (CVDs) lead the ranking of causes of death in Brazil and worldwide. At the beginning of the 20th century, they were responsible for $10 \%$ of world deaths, however, in 2013, they already accounted for $31.5 \%$. In Brazil, data from the World Health Organization (WHO), point out that CVDs caused $31 \%$ of deaths in the country in 2014, following the world average. Purpose: to evaluate the level of physical activity of patients in the preoperative period of cardiac surgery through the application of the questionnaire. Methods: The Baecke questionnaire was used as a research tool in 20 cardiac patients, referred for surgery electively, aged 30 years or over, of both sexes. The level of physical activity of these patients was analyzed from the answers they gave to two variables: Physical Leisure Exercises (EFL); and Leisure and Locomotion Activities (ALL). The evaluation of these quantitative variables was performed using Pearson's correlation. As a result, it was noticed that $60 \%$ of the patients were male, with an average age of $53 \pm 10$ years. The assessment of the interdependence between the EFL and ALL indicators performed by Pearson's linear correlation resulted in the $p$-value $<0.0001$, which is a highly significant correlation coefficient between $r=0.8648$, indicating a strong correlation between the EFL and ALL. Conclusion: Patients in the preoperative period of cardiac surgery are mostly men over 50 years old who have a good level of physical fitness. 


\section{INTRODUÇÃO}

As doenças cardiovasculares constituem a maior de todas as endemias do século XX nos países ocidentais desenvolvidos, sendo considerada epidemia progressiva o aumento da incidência do infarto agudo do miocárdio nesses países. Esse fato vem acontecendo também atualmente nos países emergentes, para as quais apontam as doenças cardiovasculares, incluindo as cerebrovasculares, ocupando o primeiro lugar ou o segundo lugar como causa de morte'.

As doenças cardiovasculares geram o maior custo referente a internações hospitalares no sistema de saúde nacional. Em 2007, 12,7\% das hospitalizações não relacionadas a gestações e 27,4\% das internações de indivíduos de 60 anos ou mais foram causadas por doenças cardiovasculares. É importante notar a carga da insuficiência cardíaca congestiva: em pessoas com mais de 60 anos é a causa mais comum de internação hospitalar; em pessoas acima de 80 anos, causa $27 \%$ das internações em mulheres e $33 \%$ em homens ${ }^{2}$.

Segundo a organização mundial da saúde (OMS), as doenças cardiovasculares são as principais causas de morte no mundo e a cada ano morrem mais pessoas por elas do que por qualquer outra causa, se calcula que em 2008 morreram cerca de 17,3 milhões de pessoas, o que representa cerca de 30\% de todas as mortes registradas no mundo, sendo 7,3 milhões por cardiopatia coronariana e 6,2 milhões por AVC. Calcula-se que em 2030 morreram cerca de 23,3 milhões, sendo o AVC a principal causa de morte, o mesmo pode ser prevenido atuando sobre os fatores de risco que são: fumo, obesidade, inatividade física, hipertensão arterial, diabetes e aumento do colesterol ${ }^{3}$.

Em 2005, as doenças cardiovasculares DCVs foram responsáveis por $28,2 \%$ dos óbitos, principal causa de morte no país. As doenças cerebrovasculares e as doenças isquêmicas do coração se destacaram como as mais frequentes causas de mortalidade por DCV, com $31,7 \%$ e $29,9 \%$, respectivamente ${ }^{4}$.

Nos últimos anos originou-se uma busca incessante pelos fatores de risco (FR) relacionados ao desenvolvimento das DCVs. Visto que a genética e a idade tenham grande importância nesta evolução, grande parte dos outros FR pode ser influenciada também por modificações no estilo de vida, com base na pratica de exercícios físicos, de forma a reduzir os eventos cardiovasculares e aumentar a sobrevida em pacientes portadores ou em risco de DCVs ${ }^{5}$.

O presente estudo também possui o objetivo de estudar um possível fator de risco das DCV'S, analisando em que medida a falta de atividade física pode ser a causadora das doenças cardiovasculares, analisando o nível de atividade física de pacientes cardiopatas no pré-operatório da cirurgia cardíaca.

Para isso, será analisado, através do questionário de Baecke, quais dos pacientes praticavam atividades físicas do lazer (EFL) e atividades física de lazer e locomoção (ALL). As EFL são aquelas que atuam como mecanismos compensatórios frente ao estresse, à angustia, à ansiedade, agindo como aliviadores de tensões, renovando as energias e proporcionando prazer ${ }^{6}$. Já as ALL referem-se às atividades de assistir à televisão (atividade sedentária), caminhar, andar de bicicleta e uma última questão sobre os minutos por dia em atividades de locomoção (caminhar ou uso de 
bicicleta para ir e voltar do trabalho, escola ou compras). Este método foi escolhido devido ao seu fácil entendimento e preenchimento, que é estruturado de forma quali-quantitativa, e vem sendo utilizado globalmente, em pesquisas com contextos mundiais ${ }^{7}$.

\section{MÉTODOS}

A pesquisa iniciou somente após a aprovação do Comitê de Ética e Pesquisa (CEP) envolvendo seres humanos da instituição estudada, unidade órgão Centro de Ciências Biológicas da Saúde e se deu conforme a Resolução no. 466/12 do Conselho Nacional de Saúde, após aprovação sob o parecer do processo №58225016.1.0000.0016, respeitando a respeitou a resolução de n 580/2018 e a de n 466/12.

Trata-se de um estudo transversal, analítico de caráter quantitativo. Para o presente estudo, foram selecionados, na primeira fase da pesquisa, 100 pacientes cardiopatas, em fase pré-operatória e que se encontravam internados nas clínicas cardiológica e cirúrgica de um hospital de referência em Belém. No entanto, desses 100 pacientes da primeira fase, apenas 20 seguiram com o estudo, pois se encaixavam nos seguintes critérios: a) os pacientes estavam selecionados para cirurgia eletiva; b) possuíam faixa etária igual ou acima de 30 anos; c) encontravam-se cognitivamente preservados sendo foram avaliados através da escala de Glasgow, que considera três fatores principais e determina uma pontuação de acordo com o nível de consciência apontada em cada um desses casos, que são a abertura ocular, a resposta verbal e a resposta motora ${ }^{8}$ d) estavam hemodinamicamente estáveis, com pressão arterial <140mmHg x 90mmHg.

Todos os pacientes que participaram da pesquisa foram elucidados e anteriormente assinaram o Termo de Consentimento Livre e Esclarecido (TCLE). Não fizeram parte desse estudo pacientes em pré-operatório que estavam na Unidade de Terapia Intensiva (UTI), na porta de entrada do Serviço de Atendimento e triagem (SAT), com síndrome coronariana aguda concomitância de cardiopatia congênita ou de doenças terminais, com angina instável, com doenças neurológicas e traumato-ortópedicas, que na fase de repouso ou na execução do teste apresentaram alterações hemodinâmicas significativas ou qualquer outra condição que contra indique a prática de atividade física.

Os participantes desse estudo foram submetidos à aplicação do questionário de Baecke, composto de 16 questões - contendo perguntas abertas e fechadas - que investiga a atividade física habitual de um indivíduo nos últimos

12 meses. É um questionário de fácil aplicação e entendimento, sendo proposto em escala quantitativa e abordando magnitudes como atividade física ocupacional, exercícios físicos no lazer (EFL), atividades físicas de lazer e locomoção $(\mathrm{ALL})^{9}$. Com relação aos EFL, buscou-se analisar a prática regular de exercícios físicos, que pode ser mensurada conforme seu gasto enérgico em rápida, moderada ou vigorosa, a partir de perguntas sobre a duração e a frequência da atividade. No caso das ALL, as perguntas são sobre atividades sedentárias - como assistir televisão - sobre caminhar, andar de bicicleta e sobre a duração diária das atividades de locomoção ${ }^{10}$. 
Foram aplicados métodos estatísticos descritivos e inferenciais. As variáveis qualitativas foram apresentadas por distribuições de frequências absolutas e relativas. Já as variáveis quantitativas foram apresentadas por medidas de tendência central e de variação, onde foi aplicado o teste de Shapiro-Wilk para avaliar a normalidade das variáveis quantitativas. A avaliação da correspondência entre as variáveis quantitativas (EFL e ALL) foi realizada pela Correlação de Pearson e a ANOVA com pós-teste de Tukey foi usada para comparar os dados deste estudo com outros estudos já publicados. Foi previamente fixado o nível de significância alfa $=0.05$ (Erro alfa $=5 \%$ ) para rejeição da hipótese de nulidade. 0 processamento estatístico foi realizado nos softwares SAM (Statistical Analysis Model) versão 2.0 e BioEstat versão 5.3.

\section{RESULTADOS}

O estudo avaliou 20 pacientes cardiopatas, internados nas clínicas cardiológica e cirúrgica (enfermarias) da FHCGV. Todos estavam dentro do critério de inclusão. Com relação a idade dos pacientes, $70 \%$ tinham acima dos 50 anos (Tabela 1). A idade variou de 32 a 76 anos, com média de 53 anos e desvio padrão de 10 anos. Já se tratando do sexo dos pacientes, 60\% deles eram do sexo masculino, conforme mostrado na Figura 2.

Tabela 1 - Distribuição da faixa etária de n=20 pacientes cardiopatas internados no FHCGV.

\begin{tabular}{lcl}
\hline Faixa Etária (anos) & Número de pacientes & $\%$ \\
\hline 30 a 39 & 2 & 10.0 \\
40 a 49 & 4 & 20.0 \\
50 a 59 & 10 & 50.0 \\
60 ou mais & 4 & 20.0 \\
\hline
\end{tabular}

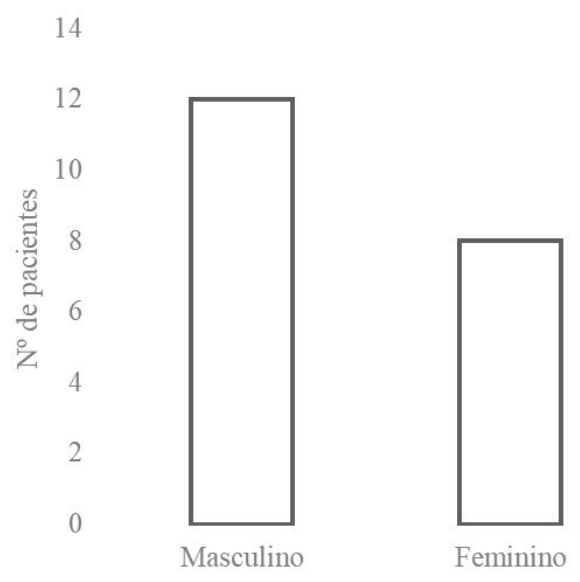

Figura 1 - Distribuição do sexo de $n=20$ pacientes cardiopatas internados no FHCGV. 
Com o auxílio do questionário de Baecke, pode-se analisar duas principais variáveis, o nível de EFL e o nível de ALL. De acordo com a resposta dos pacientes, o questionário contabiliza uma pontuação que vai 0, o mínimo, a 4 pontos, o máximo a ser alcançado em cada variável, e que pode ser classificada dentro de cinco grupos. Verificou-se, então, que $75 \%$ dos pacientes obtiveram uma pontuação boa ou ótima para EFL e que $70 \%$ alcançaram a classificação boa ou ótima para ALL. Somado a isso, nenhum dos pacientes negou a prática de exercícios e de atividades. Esses dados podem ser verificados na Tabela 2.

Tabela 2 - Classificação entre os indicadores BAECKE EFL e ALL para n=20 pacientes cardiopatas internados no HCGV.

\begin{tabular}{llllll}
\hline & BAECKE & EFL & \multicolumn{3}{c}{ ALL } \\
Pontuação & Classificação & N & $\%$ & N & $\%$ \\
\hline 0 a 0.8 & Muito baixo & 0 & 0.0 & 0 & 0.0 \\
0.8 a 1.6 & Baixo & 0 & 0.0 & 0 & 0.0 \\
1.6 a 2.4 & Regular & 5 & 25 & 6 & 30 \\
2.4 a 3.2 & Bom & 7 & 35 & 8 & 40 \\
3.2 a 4.0 & Ótimo & 8 & 40 & 6 & 30 \\
& Total & 20 & 100 & 20 & 100 \\
\hline
\end{tabular}

EFL - Exercícios Físicos de Lazer; ALL - Atividades de Lazer e Locomoção; N - número.

Ademais, também foram somadas as pontuações de EFL e ALL, resultando em um ET (Escore Total), exposto na Tabela 3. Pode-se verificar medidas de tendência central e de dispersão desta variável, ficando, então, evidente uma diferença de 3.87 pontos entre o paciente de maior pontuação e o de menor pontuação. Além disso, percebe-se que a pontuação máxima e mínima de ALL foi maior quando comparada com a de EFL.

Tabela 3 - Avaliação da atividade física pelo instrumento BAECKE, para n=20 pacientes cardiopatas internados no FHCGV.

\begin{tabular}{lllll}
\hline & \multicolumn{2}{l}{ BAECKE } & & \\
Estatísticas de resumo & EFL $_{1}$ & & ALL $_{2}$ & ET $_{3}$ \\
\hline Mínimo & 1.60 & 1.80 & 3.40 & \\
Máximo & 3.47 & 3.80 & 7.27 & \\
Mediana & 2.80 & 2.70 & 5.50 & \\
Primeiro Quartil & 2.54 & 2.20 & 4.74 \\
Terceiro Quartil & 3.20 & 3.20 & 6.40 \\
Média Aritmética & 2.77 & 2.74 & 5.51 \\
Desvio Padrão & 0.59 & 0.58 & 1.15 \\
Coeficiente de Variação & $21.3 \%$ & $21.2 \%$ & $20.9 \%$ & \\
\hline
\end{tabular}

${ }_{1}$ EFL - Exercícios Físicos de Lazer; ${ }_{2} A L L$ - Atividades de Lazer e Locomoção; ${ }_{3} E T$ - Escore; Total de atividade física: EFL + ALL. 
Por último, avaliou-se a interdependência entre os indicadores EFL e ALL, a qual foi realizada pela Correlação linear de Pearson que resultou no p-valor <0,0001, o qual é altamente significante, com Coeficiente de Correlação entre $r=0.8648$, indicando que existe uma forte e positiva correlação entre o EFL e o ALL, como mostra a Figura 2.

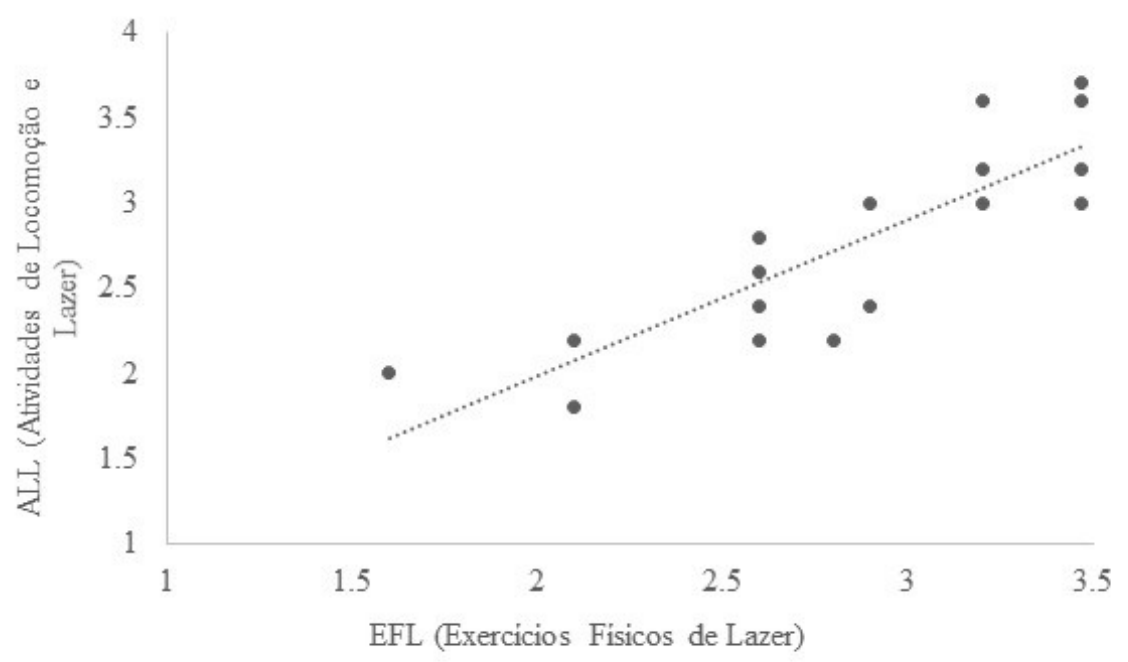

Figura 2 - Correlação entre os indicadores BAECK EFL e ALL de n=17 pacientes cardiopatas internados no FHCGV*

* Coeficiente de Correlação entre EFL e ALL = 0.8648

\section{DISCUSSÃO}

Tendo em vista que as respostas da EFL levam em consideração a intensidade do exercício, é esperado que os participantes, principalmente os mais velhos, não sejam estimulados a praticar exercícios vigorosos. Os profissionais devem ser cautelosos em prescrever atividade física, em particular atividade não supervisionada para pacientes com doença cardiovascular conhecida devido a preocupações com o risco de eventos cardiovasculares adversos. Atividades intensas podem causar microisquemias em algumas áreas dos miocárdios, o que pode originar microfibroses que servem de gatilho para 0 aparecimento de arritmias cardíacas ${ }^{11}$. Estas arritmias, então, são capazes de virarem fibrilações ventriculares que podem causar a morte do cardiopata ${ }^{12}$. Logo, fica claro que a atividade física possui benefícios, porém deve ser realizada com cautela, respeitando a história clínica e o perfil de cada paciente.

Recomenda-se, então que a prática de atividades físicas para esses pacientes seja supervisionada e limitada. O treinamento físico deve ser iniciado em uma intensidade baixa (mais) e aumentado gradualmente ao longo do tempo. Os exercícios de resistência devem ser complementados por exercícios de resistência 2 vezes por semana em intensidade moderada. 
Quanto à análise do ET, relacionando-o com as classificações de pontuação do questionário de Baecke, percebese que boa parte dos participantes da pesquisa tiveram um nível de atividade física bom, além de nenhum ter sido mal classificado com pontuação baixa ou muito baixo. Essa realidade, se mantida, poderá agregar valor na reabilitação cardíaca dos pacientes durante o pós operatório. Sabe-se que os exercícios físicos diminuem a taxa de reincidência de internação hospitalar, além de diminuir a chance de mortalidade cardíaca (4). Somado a isso, em pacientes que realizaram transplante cardíaco, os exercícios físicos após as cirurgias geram melhoras cardiorrespiratórias, endoteliais, metabólicas e morfológicas, sendo recomendados pelas diretrizes da American Heart Association e da American College of Sports Medicine ${ }^{13}$.

\section{CONSIDERAÇÕES FINAIS}

O indivíduos com doenças cardiovasculares, apesar de mostrarem-se fisicamente ativas parte realizou exercício físico de forma regular e parte como atividade de lazer. Sabendo que para a segurança dos cardiopatas o exercício supervisionado é o mais indicado, esse estudo sinaliza que parte dos cardiopatas realiza atividade de lazer, uma atividade de forma não regular.

\section{REFERÊNCIAS}

1. CUPPARI, L. Nutrição clínica no adulto. 2. ed. Barueri, SP: Manole, 2005.

2. INSTITUTO BRASILEIRO DE GEOGRAFIA E ESTATÍSTICA - IBGE.Indicadores Sociodemográficos e de Saúde no Brasil 2009. Estudos e Pesquisas Informação Demográfica e Socioeconômica, número 25, 2009.

3. SANTOS, RD; GAGLIARDI, ACM; XAVIER, HT;MAGNONI, CD; CASSANI, R;LOTTENBERG, AM et al. Sociedade Brasileira de Cardiologia. I Diretriz sobre o consumo de Gorduras e Saúde Cardiovascular. Arquivo Brasileiro de Cardiologia.v. 100, n. 3. p. 1-40, 2013.

4. BRASIL. Ministério da Saúde. 2008 . DATASUS: informações de saúde, mortalidade-Brasil. Disponível em:<http://w3.datasus.gov.br/datasus/datasus.php>. Acesso em ago. de 2020. 
5. RIQUE, ABR; SOARES, EA; MEIRELLES, CM. Nutrição e exercício na prevenção de doenças cardiovasculares. Revista brasileira de medicina e Esporte, Rio de Janeiro, v. 8, n. 6.nov. /dez. 2002.

6. RIOS. Luzana Cirqueira; ALMEIDA, Maria Guimaraes de; ROCHA, Saulo Vasconcelos; ARAUJO, Tania Maria; PINHO, Paloma de Sousa. Atividades Fisicas de Lazer e Trastornos mentais comuns em jovens de Feira de Santana.

7. DELVAUX K, Lefevre J, PHILIPPAERTS R, DEQUEKER J, THOMIS M, VANREUSEL B, et al. Bone mass and lifetime physical activity in flemish males: a 27-year followup study. Med Sci Sports Exerc 2001;33:1868-75

8. MCNAMARA, Damian. Escala de coma de Glasgow atualização esclarecedora. 2018.

9. Almeida MCB, Ribeiro JLP. Adaptação do Habitual Physical Activity Questionnaire (Baecke), versão modificada, para a população portuguesa. Revista de Enfermagem Referência. 2014;27-36. Available from: http://dx.doi. $\operatorname{org} / 10.12707 / \mathrm{RIII} 1303$

10. Florindo AA, Do M, Dias De Oliveira Latorre R, Constante Jaime P, Tanaka T, Augusto De Freitas Zerbini C. Metodologia para a avaliação da atividade física habitual em homens com 50 anos ou mais. Revista Saúde Pública. 2004;307-21. Available from: www.fsp.usp.br/rsp

11. Matsudo VKR, Santos M dos, Beltran DCG, Guedes J da S. Exercício: quanto mais, melhor? Diagnósico e Trat. 2019;24:67-9. Available from: https://health.gov/paguidelines/second-edition/pdf/Physical_

12. Perrier-Melo RJ, Figueira FAM dos S, Guimarães GV, Guimarães GV. High-Intensity Interval Training in Heart Transplant Recipients: A Systematic Review with Meta-Analysis. Arq Bras Cardiol. 2018;110. No 2:188-94.

13. MARANHAO NETO, Geraldo de Albuquerque et al. VALIDADE DIAGNÓSTICA DO QUESTIONÁRIO DE TRIAGEM DO AMERICAN COLLEGE OF SPORTS MEDICINE/AMERICAN HEART ASSOCIATION. J. Phys. Educ. [online]. 2019, vol.30. 